\title{
Frequency characteristics of perforation and fracturing induced microseismic events recorded by downhole and surface arrays
}

\author{
Ruizhao Yang \\ College of Geoscience \& Surveying Engineering \\ China University of Mining \& Technology at Beijing \\ Beijing, China \\ yrz@cumtb.edu.cn
}

\author{
Zhengguang Zhao \\ Beijing GeoSun Energy Tech., Ltd \\ Beijing, China \\ zhzhg.uq@gmail.com
}

\begin{abstract}
Surface microseismic monitoring of hydraulic fracturing is now becoming more and more popular in the production of unconventional hydrocarbons. The frequency analysis of surface detected microseismic event and comparison with perforation event and downhole recorded event may lead to deeper understanding of the frequency variations of microseismic events and contribute to the development of innovative filtering techniques. In this paper, two microseismic events detected by surface arrays, one microseismic event and one perforation event recorded by downhole geophones are presented. $\mathrm{S}$-transform is used to derive the time frequency representations of these events. The frequency contents of these three types of microseismic events are characterized and compared with each other.
\end{abstract}

Keywords - microseismic event; perforation; downhole array; surface array; time frequency spectrum; $S$-transform

\section{INTRODUCTION}

Since the resolution of source location is a function of the peak frequency of the observed direct $\mathrm{P}$ - and $\mathrm{S}$ - waves of microseismic event,understanding the frequency characteristics of the observed signals plays a crucial role in locating microseismic events. It's reported that microseismic events observed by downhole monitoring tools typically have peak frequencies above several hundred hertz ${ }^{[1,2]}$, while events observed on surface networks are usually dominated by amplitudes with peak frequencies below $50 \mathrm{~Hz}^{[3]}$. Eisner et al(2013) have also explored the peak frequency of direct waves for microseismic event and found that observed peak frequency (also known as the useful signal) can be described by a combination of the source radiation and the global absorption factor ${ }^{[4]}$. Compared to North America where the unconventional hydrocarbon reservoirs have less TVD depth, China has a very different geological settings with respect to the unconventional resources especially for tight gas and shale gas, which may lead to large discrepancy in frequency content of microseismic event recorded by both surface and downhole array.

In this study, we show three cases from domestic oil and gas industry, in which the time frequency representations of perforation and fracturing induced microseismic events recorded by downhole and surface arrays are obtained using Stransform.

\section{THEORY AND METHOD}

Microseismic signals are actually non-stationary signals. In non-stationary signal processing field, the Short-Time Fourier Transform(STFT), Continuous Wavelet Transform(CWT) and S-transform are the most common way to derive the timefrequency representation for the non-stationary signals. The popular methods of STFT and Wavelet analysis have limitations in representing close frequencies and dealing with fast varying instantaneous frequencies and this is often the nature of microseismic signals ${ }^{[5]}$.

The S-transform, which is first proposed by R.G. Stockwell in 1996, is unique in that it provides frequency-dependent resolution while maintaining a direct relationship with the Fourier spectrum ${ }^{[6]}$. It is an extension of the ideas of the STFT and is based on a moving and scalable localizing Gaussian window. It proves to have a good performance of noise reduction as well as desirable characteristics that are absent in $\mathrm{CWT}^{[7]}$. Therefore, the S-transform is a more preferable tool to track resonant frequencies and provide a detailed timefrequency representation. There are several ways to represent the idea of the S transform. In here, S-transform is derived as the phase correction of the CWT with window being the Gaussian function.

The definition of CWT is

$$
W(\tau, d)=\int_{-\infty}^{+\infty} x(t) \omega(t-\tau, d) d t
$$

$\omega(t, d)$ is wavelet mother function. The dilation factor $d$ controls the width of wavelet basis and the frequency resolution.

The mother wavelet is defined as

$$
w(t, f)=\frac{|f|}{\sqrt{2 \pi}} e^{-\frac{t^{2} f^{2}}{2}} e^{-j 2 \pi f t}
$$


The S-transform of function $x(t)$ is defined as a CWT with a special mother wavelet multiplied by the phase factor

$$
S(t, f)=e^{j 2 \pi f \tau} \int_{-\infty}^{+\infty} x(t) \omega(t-\tau, f) d t
$$

The dialation factor $d$ is the inverse of the frequency $f$. And the wavelet in (1) doesn't satisfy the admissibility condition. So the S-transform is

$$
S(\tau, f)=\int_{-\infty}^{+\infty} x(t) \frac{|f|}{\sqrt{2 \pi}} e^{-\frac{(\tau-t)^{2} f^{2}}{2}} e^{-j 2 \pi f t} d t
$$

\section{CASE STUdiES}

\section{A. Microseismic Events Detected at Surface}

Here we present two microseismic events recorded by surface broadband seismograph stations $(4.5 \mathrm{~Hz} 3 \mathrm{C})$ for monitoring hydraulic fractures during the stimulations of two domestic gas wells, i.e. TX1 vertical well located in Cengong, Guizhou and SS103H horizontal well located in Songliao Basin

As for TX1 well, a total of 33 visible events(Fig.1) are detected by the surface array consisting of 48 stations during the single one stage stimulation of shale gas reservoir at a TVD of $1800 \mathrm{~m}$.

However, only a total of 41 visible microseismic events are verified out of over 70 candidate events after filtering and location during all 15 stages of hydraulic fracturing of SS103H well, of which the target tight gas reservoir's lithology is intermediate igneous rock with a TVD of $3140 \mathrm{~m}$. The scarcity of microseismicity is likely because the reservoir is deeper and there is also one nearly $100 \mathrm{~m}$ weathering layer with a very low velocity, which lead to more amplitude attenuation of $\mathrm{P}$ and $\mathrm{S}$ waves and make them hard to be detected at surface.

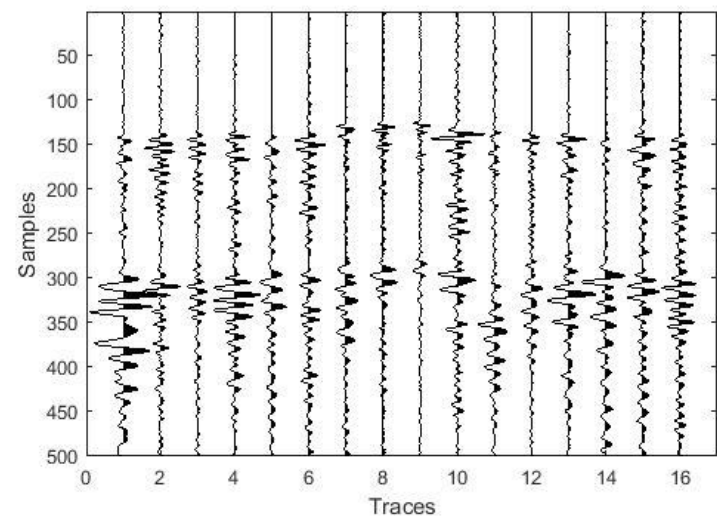

Fig.1. P- and S- waveforms of one visible microseismic event shown on vertical components of $163 \mathrm{C}$ stations deployed for monitoring the stimulation of TX1 well.

Examples of waveforms of one hydraulic fracturing induced microseismic event recorded during the stimulation of
TX1 well together with time-frequency analyses are shown in Fig.2. In this case, the S-wave arrivals have high amplitudes on all three components. Due to the incidence angle and station azimuth, the amplitude of direct $\mathrm{P}$-wave on the vertical component are much larger than those on horizontal components, but it's still possible to discern P-wave arrivals on the horizontal components from the background noise. The time frequency analysis for this specific event using $\mathrm{S}$ transform shows that the frequency content of P-wave is predominantly concentrated above $40 \mathrm{~Hz}$, with a peak frequency up to $70 \mathrm{~Hz}$. S-wave's frequency is around $30 \mathrm{~Hz}$, which is lower than that of P-wave.

Fig.3 shows the recorded waveforms and corresponding spectrograms of one hydraulic fracturing induced microseismic event recorded during the stimulation of SS103H well. In this case, the frequency bandwidth of $\mathrm{P}$-wave arrival is in the range of 30 to $50 \mathrm{~Hz}$, while the frequency of $\mathrm{S}$ - wave arrival is significantly lower and only about $8 \mathrm{~Hz}$. Compared to the microseismic signals recorded for TX1 well, the frequency contents of both P- and S-wave arrivals are much lower. One possible reason is, as mentioned above, this low frequency is resulted from the thick low-velocity near-surface layer which has greatly attenuated the S-wave frequency.
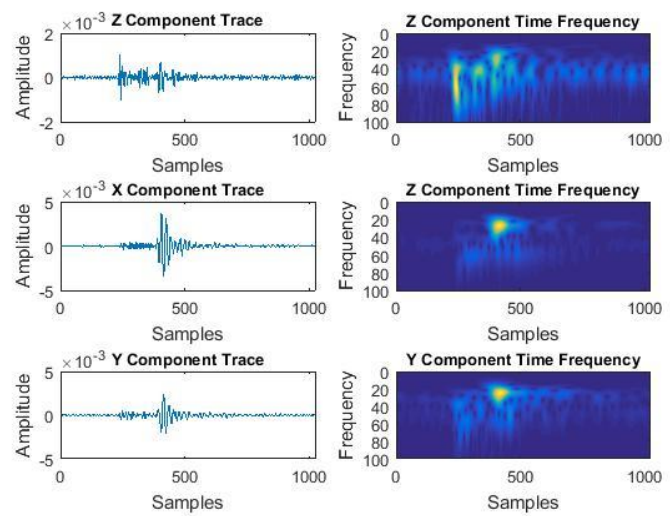

Fig.2. Examples of recorded waveforms and their corresponding spectrograms of one microseismic event detected during the stimulation of TX1 well. The spectrograms are obtained using the S-transform.
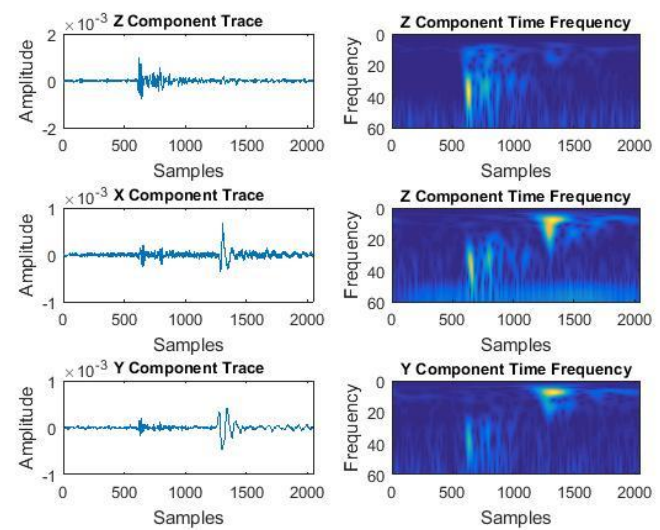

Fig.3. Examples of recorded waveforms and their corresponding spectrograms of one microseismic event detected during the stimulation of SS103H well. The spectrograms are obtained using the S-transform. 

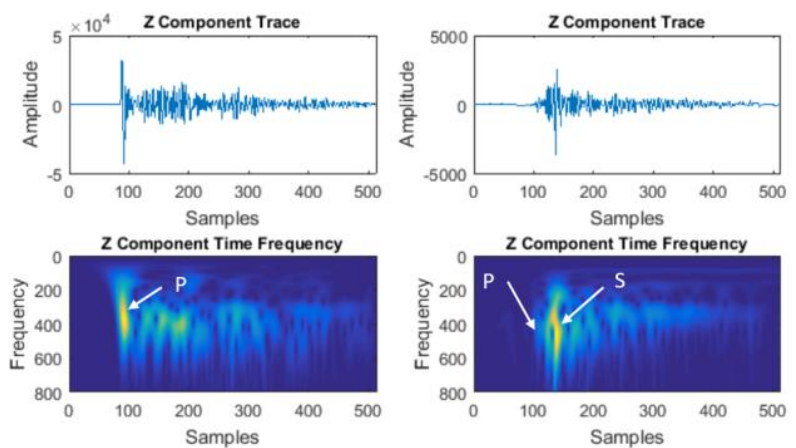

Fig.4. Examples of perforation shot event and microseismic event. Upper panels: recorded waveforms of perforation shot event and microseismic event from left to right respectively. Lower panels: corresponding spectrograms obtained using the S-transform.

\section{B. Microseismic Events Detected by Downhole Array}

Examples of waveforms recorded from perforation shots and hydraulic fracturing induced microseismic event together with time-frequency analyses are shown in Fig.4. The perforation depth is around $2000 \mathrm{~m}$ TVD, with a distance of $800 \mathrm{~m}$ from the stimulated well.

For perforation event, as expected due to the volumetric nature of explosive sources, the waveforms are dominated by the direct P-wave, with a peak frequency of around $400 \mathrm{~Hz}$. It also can be found that the high-frequency content diminishes with distance due to the effects of anelastic attenuation. In the case of hydraulic fracturing induced microseismic event, the Swave arrival has the highest amplitudes while $\mathrm{P}$-wave arrival is hard to be distinguished from the background noise. The frequency content of $\mathrm{S}$-wave is predominantly concentrated above $200 \mathrm{~Hz}$ and the bandwidth decreases with distance due to the effects of attenuation.

\section{CONCLUSIONS}

This study shows that great discrepancy exist between the peak frequencies of microseismic events recorded by surface and downhole arrays, i.e. surface detected events usually have lower frequencies (around $30-70 \mathrm{~Hz}$ of $\mathrm{P}$-wave and $8-30 \mathrm{~Hz}$ of $\mathrm{S}$-wave) while perforation and hydraulic fracturing induced events observed on downhole geophones typically have a higher frequency content which is predominantly concentrated above $200 \mathrm{~Hz}$

Comparison of two surface detected microseismic event show that attenuation resulted from thick near-surface lowvelocity layer has further decreased the signal frequency. This may explain why the frequency of microseismic event detected by surface array deployed in Northeastern China is usually much lower than those recorded in Southern China, where a number of emerging shale gas production area are located.

\section{ACKNOWLEDGMENT}

We would like to thank Daqing Oil Field, PetroChina and Tongren Zhongneng Gas Company for the permission of release of the datasets used in this study.

\section{REFERENCES}

[1] J.T. Rutledge and W.S. Phillips, "Hydraulic stimulation of natural fractures as revealed by induced microearthquakes, Carthage Cotton Valley gas field, east Texas," Geophysics, 2003, 68(2), pp.441-452, doi: $10.1190 / 1.1567214$

[2] I. Das and M.D. Zoback, "Long-period, longduration seismic events during hydraulic fracture stimulation of a shale gas reservoir," The Leading Edge, 2011, 30(7), pp.778-786, doi: 10.1190/1.3609093.

[3] P. Duncan and L. Eisner, "Reservoir characterization using surface microseismic monitoring," Geophysics, 2010, 75, pp.75A139-75A146, doi: $10.1190 / 1.3467760$

[4] L. Eisner, D. Gei, M. Hallo, I. Opršal and M.Y. Ali., "The peak frequency of direct waves for microseismic events," Geophysics, 2013, 78(6), pp.a45-a49

[5] R.H. Herrera, J.B. Tary and M. van der Baan, "Time-frequency representation of microseismic signals using the synchrosqueezing transform," GeoConvention 2013: Integration, CSEG, Expanded Abstracts

[6] R.G. Stockwell, L. Mansinha and R.P. Lowe, "Localization of the complex spectrum: The S transform," IEEE Transactions on Signal Processing, 1996, 44(4), pp.998-1001.

[7] Y. Lin, X. Xu, B. Li and J. Pang, "Time-frequency analysis based on the S-transform," International Journal of Signal Processing, Image Processing and Pattern Recognition, 2013, 6, pp.245-254. 